\title{
Some reflections on IPv6 adoption in Australia
}

\author{
Peter Dell $^{1 *}$, Christopher Kwong ${ }^{2}$, Ying Liu ${ }^{1}$ \\ 1 Curtin University of Technology, Perth, Australia \\ 2 Australian Government, Canberra, Australia
}

\begin{abstract}
IPv6 is an inevitable upgrade to Internet Protocol (IP), the underlying protocol upon which the Internet is based, and solves many problems with the existing technology including limited address space, performance and security. Although IPv6 has been supported by products from major vendors for many years, adoption has been practically non-existent. Meanwhile, the Internet continues to grow and the size of the problem increases.

This unsatisfactory situation is a consequence of network externalities in which rational individuals have no motivation to be the "first-mover". We argue that government action is required to promote diffusion of IPv6, but note that this is difficult and may be unlikely in the absence of a business case. Making the case for IPv6 should not be problematic given the range of "hidden" costs of retaining the status quo; however, there has been no systematic effort to assess such costs. We thus recommend a number of directions for future research to address this deficiency.
\end{abstract}

\section{INTRODUCTION}

The Internet is based on a communications protocol called IP (Internet Protocol) and since 1983 the Internet has run on IP version 4 (IPv4). Design decisions made during the 1970s were based on assumptions about the size of the Internet that are now no longer appropriate; even by the late 1980s the need to revise IP had been recognised (Loshin, 2004).

The most widely-known flaw in IPv4 is the size of its address space, which is theoretically large enough to contain 4.3 billion addresses, and in practical terms is far fewer due to overheads in address allocation schemes. While these numbers may seem large, it is clearly not sufficient to cater for the massive growth in Internet connected devices that began in the 1990s and is likely to continue for many years to come. A related weakness is that the size of the routing tables in the IPv4 Internet backbone has grown at an alarming rate. This causes delays in processing packets as they traverse each router, thus contributing to increased latency in the network as a whole.

Workarounds such as Network Address Translation (NAT) and Classless InterDomain Routing (CIDR) were developed during the 1990s to slow the rate at which IPv4 address space is consumed, and have succeeded in slowing address space consumption from an exponential to a roughly linear rate. However, these approaches

\footnotetext{
* Corresponding author. Email P.T.Dell@curtin.edu.au.
} 
were not intended to be long-term solutions; NAT in particular imposes significant problems, discussed below.

Further, security was not a consideration when IPv4 was designed and has no underlying authentication or encryption mechanisms. While workarounds have been developed to allow reasonably secure transactions, these are not universal and the prevalence of Denial of Service attacks and spam email are testament to the inadequacy of IPv4.

The only realistic long-term solution is to upgrade IP itself. IP version 6 (IPv6) was recommended the IETF in 1994 as a replacement to IPv4 and was designed to operate smoothly in conditions ranging from extremely high performance to low bandwidth networks, such as wireless networks. It solves problems with address space by handling up to $3.4 \times 10^{38}$ addresses - easily enough to handle continued exponential growth of the Internet due to connection of mobile, peer-to-peer and 'smart home' devices.

IPv6 also addresses security by including authentication, integrity and confidentiality services as standard. IPv6 also includes a number of benefits such as autoconfiguration, improved routing, and built-in support for Quality of Service (QoS).

\section{IPV6 TRANSITION}

Given the problems with IPv4 and the prospect of imminent exhaustion of IPv4 address space it is desirable to encourage migration to IPv6 as soon as reasonably possible, and given the size of the transition this will take many years. This will inevitably result in some parts of the network having migrated to IPv6 while others are still IPv4. The inevitable question is hence where to begin the migration: at the core, or at the edge of the network.

Beginning the transition at the core is considered problematic because it requires translation from IPv4 to IPv6 be conducted at all entry points to the migrated Internet backbone. This is a huge task, and it is very unlikely that such an approach could be implemented without disrupting connectivity. Hence, it is often recommended that transition should begin from the edge of the network, rather than the core (Tatipamula et al., 2004). This approach effectively seeks to create "islands" of IPv6 connected by tunnels over an IPv4 backbone, and continues until IPv6 traffic reaches sufficient volumes to warrant deploying both IPv4 and IPv6 over the backbone. Eventually, as the volume of IPv4 traffic withers, IPv4 is tunnelled over IPv6.

Unfortunately, historically it has proven extremely difficult to persuade organisations at the edge of the network to adopt IPv6, forming a significant barrier to the transition to IPv6. A prime reason for this is a common belief that NAT is a sufficient solution for at least the medium term. However, NAT is not ideal for a number of reasons.

First, incoming connectivity is problematic with NAT, as it hides internal addresses from the rest of the world. This can be overcome with Port Address Translation (PAT), in which NAT redirects traffic from specific port numbers to internal servers, but this effectively limits an organisation to having a single server of any type. For 
example, PAT may be used to redirect incoming connections to port 80 to a web server, but the organisation cannot easily install multiple public web servers.

Another problem with NAT is that some protocols have difficulty traversing a NAT. This is frequently an issue with UDP-based protocols, including many newer applications such as Voice over IP. Further, many peer-to-peer applications and security standards such as Kerberos, IPsec and proprietary VPNs frequently experience incompatibility problems with NAT. Workarounds such as STUN (Simple Traversal of UDP through NAT) or ALGs (Application Layer Gateways) exist for this problem, but these do not work with all applications and necessitate extra costs for the installation and maintenance of additional servers.

Entrenching the belief that NAT is a suitable solution to the problems with IPv4 is the belief that it has minimal Total Cost of Ownership (TCO) (Liu, 2006). Yet NAT actually drives up network TCO, as new services and applications will constantly necessitate otherwise unnecessary workarounds (Cooper and Yen, 2005).

A further barrier to IPv6 adoption is the perception that IPv6 is immature. IPv6 standards expected to evolve throughout the transition period; this is not particularly problematic and is actually a normal evolution of Internet standards. Further, base protocols are stable and secure, although many value-adding protocols are still emerging (CIO Council, 2006; c.f. Kwong, 2006).

Perhaps the biggest barrier to organisations at the Internet's edge adopting IPv6 is a reluctance to become early adopters. This results in a situation that is reminiscent of Hardin's (1968) "tragedy of the commons" (Liebowitz and Margolis, 1994). It is in each individual's best interests to avoid being an early adopter, or "first-mover", as network externalities would result in a cost to the user rather than a benefit. Yet it is in the interest of society as a whole that all users adopt the new standard. In this situation, where individuals cannot be expected to adopt the new standard of their own volition, action may be required to stimulate adoption.

One perspective on how to address this issue can perhaps be described as rather laissez-faire, in which market forces and the economics of supply and demand dictate the pace at which transition occurs. This perspective holds that it is acceptable to allow IPv4 to reach "breaking point" (e.g. exhaustion of address space), effectively turning IPv4 address space into a scarce resource for which there would be increasing demand. This would prompt organisations to migrate to IPv6 as they seek to avoid increasing costs associated with continued IPv4 use.

However, this is not an ideal outcome as it increases the size of the problem. The effort required to migrate to IPv6 increases as the Internet grows in size, and earlier migration is a smaller and easier task than later migration. New machines added to the network after migration will not require any effort to be spent migrating them since they will use the new standard by default. Thus, assuming that migration to IPv6 is inevitable, earlier migration is thus more efficient because the number of devices requiring migration is fewer. Thus, continuing to rely on IPv4 technologies such as DHCP and NAT for longer than necessary is actually a double investment, because the transition to IPv6 is inevitable (Cooper and Yen, 2005). 
The laissez-faire approach also 'prolongs the agony' associated with IPv4 use. This involves costs associated with ongoing administration and maintenance of services which are unnecessary under IPv6, such as DHCP and NAT, poor support for mobile devices, increased pressure on backbone route tables, increased security problems such as Denial of Service attacks, and so on.

Finally, creating a situation in which address space becomes increasingly expensive due to restricted supply limits the scope for growth of the Internet, and thus the development of new Internet-based services, and may even create possibilities for a black market in IPv4 address space (JPNIC, 2006).

For these reasons, it is arguably a better approach to take action to promote IPv6 adoption. This may take the form of stimulating market forces; indeed, "it is possible that society could benefit from accelerating the natural market-based adoption process" (NTIA, 2004: 59), or may involve regulation to require adoption.

\section{IPV6 PROMOTION AROUND THE WORLD}

Hovav et al. (2004) recommend a number of means to speed the diffusion of IPv6 through the Internet: sponsorship; policy setting; encouraging backwards-compatible products; and encouraging transitional and related technologies. Some of these approaches have been implemented in various countries; this section examines these efforts with a view to discerning what approaches might be feasibly implemented in Australia.

One obvious possibility to increase adoption of IPv6, or indeed any technology, is to make it mandatory, although some assert that past experience with Government OSI Profiles is a convincing argument that regulatory intervention by government will fail (e.g. Huston, 2006). We assert that while there may have been some resistance to standards perceived as being governmentally imposed on academia and industry, technical weaknesses and competition from TCP/IP doomed OSI to failure regardless of regulatory intervention (c.f. Tanenbaum, 1996). Further, transitions from analogue to digital mobile telephony and analogue to digital television have both been mandated by various governments with considerable success, so perhaps it is not possible to extrapolate from past failures with OSI to failure of IPv6 in the future.

Indeed, IPv6 has been mandated in other countries with success - the South Korean Ministry of Information and Communications, for example, has a policy (Policy IT839) mandating civilian use of IPv6 by 2010 (Hill, 2006), and IPv6 has been deployed since 2004 in government networks such as the postal office, universities, ministry of defence and local government agencies (Ladid, 2006). Despite this, the South Korean government still acknowledges its support policies for IPv6 have been insufficient (Biber, 2006)

Similar government endorsement is also in place in China and Japan and as a result, ISPs in China and Japan are now adopting IPv6 extensively (Hovav and Schuff, 2005). In China, the Chinese Next Generation Internet (CNGI) Project commenced in 2002 and has been heavily supported by both government and industry, while the Japanese IPv6 Council has promoted IPv6 aggressively as a "national infrastructure 
mission" to support the government's "u-Japan" (Ubiquitous Japan) concept (Ladid, 2006).

Taiwan has also invested heavily, despite being a relative late-comer to IPv6. The Taiwan National IPv6 Program is part of the US\$1b e-Taiwan program, and aims to have 6 million broadband users using IPv6 by 2008, while government networks are planned to be IPv6 ready by the end of 2007 (Ladid, 2006).

Elsewhere in Asia, the Indian Minister for IT and Communications has acknowledged the importance of IPv6 with its inclusion on a ten-point agenda for Indian IT (CIOL, 2004), and although the (extremely) ambitious goal of migration by 2006 has not eventuated, IPv6 remains a priority. The India Telecom Regulatory Authority (TRAI) has been actively promoting IPv6, and there are at least two IPv6 ISPs operating in India (Sify and VSNL/Teleglobe) (Ladid, 2006).

It is not only Asian countries that have taken steps to vigorously push IPv6. The U.S. government began working on IPv6 in 2001 via the North American IPv6 Task Force (NAv6TF), and in 2005 mandated that all federal government agencies must be IPv6capable on their backbone networks by June 2008; at the very least, agencies must be able to transfer IPv6 traffic between agency LANs and the Internet (CIO Council, 2006). Given the size of the transition task, Strauss (2005) noted that work needed to commence immediately and estimated that over $30 \%$ of agencies attempting the transition would still not be able to meet the 2008 deadline - particularly those that had only just begun planning for the transition by 2005 - further reinforcing the point that upgrading to IPv6 will be a long and drawn-out process and that it is important to start the process sooner rather than later.

In response to government initiatives at the federal level, other American ICT organisations are beginning to understand the value of IPv6. Many state and local government agencies have expressed interest in migration to IPv6 (Juniper, 2006), illustrating the efficacy of a government "push", as opposed to industry "pull", approach. And even despite this comparatively advanced state of IPv6 transition in the U.S., concerns have been expressed in the U.S. that the "[IPv6] head start of other nations will hurt the U.S." (Kirkpatrick, 2006).

In Europe, the European Commission established an IPv6 task force in January 2002 and formulated a transition road map to promote migration activities across the continent, stating that Europe's competitiveness in mobile and wireless technologies would be jeopardised if there was no clear roadmap for IPv6 transition (IPv6 Task Force, 2005). The task force has been supported by many European governments, and funding has exceeded $€ 100 \mathrm{M}$ over five years (Ladid, 2006). There has been "slow but consistent" adoption of IPv6 by ISPs in Western Europe (Hovav and Schuff, 2005) as a consequence.

\section{FUTURE IPV6 PROMOTION IN AUSTRALIA}

In contrast to many other countries, Australian adoption of IPv6 has been remarkably low, yet ICT makes a higher contribution to economic growth in Australia than other OECD countries (Biber, 2006), and many of the countries that have aggressively promoted IPv6 are major Australian trading and strategic partners (Hill, 2006). 
Further, the ability to connect a huge range of devices to the Internet, including telephones, household appliances, cars, and indeed "any device worth more than $\$ 10$ " opens up hitherto unforseen opportunities (Biber, 2006). IPv6 may also result in reduced costs to the ICT industry; Esaki (2006) notes for example that experience in Japan has shown that IPv6 contributes to easier system design and architecture and faster trouble-shooting.

Yet serious discussion about IPv6 adoption in Australia only began in 2005 (Hill, 2006), and IPv6 adoption has primarily been promoted by the IPv6 Special Interest Group within the Australian chapter of the Internet Society (ISOC-AU), a small, nonprofit organisation.

All of the examples in the previous section demonstrate that government measures to encourage IPv6 adoption can be successful, but in Australia there has been little government involvement. Further, the Australian Government currently has no intention to mandate IPv6 for government agencies (Dale, 2006), and while defence departments in other countries have lead the way in promoting IPv6 deployment in other countries, the Australian Department of Defence has set the comparatively late target date of 2013 for the transition to IPv6 ${ }^{1}$ (Richards, 2006).

Given the apparent success of government intervention in other countries, it is proposed here that action within government agencies to seed wider industry adoption and the possibility of regulatory intervention may both be reasonable in Australia and should at least be considered. Currently there are several factors that may impede such action, however, principal among which is the previously noted perceived satisfaction with IPv4 and NAT among the ICT community.

It is because of this misguided satisfaction that government intervention may be difficult for industry regulators to "sell" to the ICT industry, however, and part of the success of government measures should be addressing industry contentedness with IPv4. In particular, Liu (2006) noted a range of ICT industry perceptions of the costs of operating IPv4, characterised in many cases by lack of awareness of the costs or a belief they were not extensive. Challenging such views is an important step to ensuring acceptance of government measures to promote or mandate IPv6.

Indeed, the Australian Government has previously identified the need for a compelling business case as a key policy issue (Dale, 2006). This is a challenging issue because information on the business case for IPv6 is difficult to find, such that the only people likely to find it are those already committed to IPv6 diffusion. Hence, there is a clear need for such information to be made more widely available.

Further, business and technical cases for IPv6 are often blurred, and the majority of information available about the benefits of IPv6 is aimed at a relatively technical audience. There is a need to provide a greater business case that addresses the concerns likely to be held by policy makers and decision makers without such a technical background.

\footnotetext{
${ }^{1}$ It is noted that 2013 is somewhat later than the date by which the IANA predicts the IPv4 address space will be exhausted, which may be as early as 2010 .
} 
Still further, there is a need for debate of what might be called the 'national interest' case. Given the importance of ICT to Australian economic growth, and the efforts to accelerate adoption in major national trading partners, it may well be that it is Australia's national interest to promote adoption now. While the business case is relevant to individual organisations and is key to persuading organisations to make the transition to IPv6, debate centred on the business case alone ignores the previously identified problem in which network effects discourage being a first-mover, possibly resulting in a disadvantageous outcome overall.

For governments, the difference between business case and national interest case is essentially the difference between ICT user and ICT regulator/leader. The authors do not take a position with respect to the contribution IPv6 may make to the national interest; our purpose here is to flag the issue for discussion. The previous discussion suggests that IPv6 may be nationally significant, but no serious investigation into the consequences for Australian national interest of earlier or later adoption has been made. This argument is summarised in the following section, in which a 'call to arms' for further research is issued.

\section{CONCLUSIONS}

The current version of IP in use on the Internet, IPv4, has significant problems for which the only long-term solution is to migrate to IPv6. Considerable efforts to promote IPv6 adoption have been under way in various countries for several years, including South Korea, Japan, Taiwan, China, and the United States, and are beginning to bear fruit: IPv6 adoption is increasing, particularly in Asian countries where efforts have been the most concerted. Although all of these countries are major trading and/or strategic partners with Australia, no major efforts have been made in Australia, and there any no known major efforts on the horizon.

It is unreasonable to expect industry to make an early transition to IPv6 of its own volition due to reluctance of individual organisations to become early adopters. If this trend continues, it is likely that Australia will be a late-comer to IPv6, and while avoiding early adoption is rational at an individual level, collectively it may be contrary to the national interest.

No research has been conducted into the economic consequences to Australia of earlier or later transition of IPv6. The authors do not take a position as to which is best, but seek to highlight the need for wider debate about whether stimulating IPv6 is necessary, and if so, how it should be done. Thus, the following specific issues are identified for further discussion and research:

- The impact IPv6 will have on the contribution of ICT to economic growth

- The impact IPv6 will have on other issues of importance to the national interest

- The Total Cost of Ownership of IPv4

It is the authors' hope that addressing these questions will assist a sound transition to IPv6. 


\section{REFERENCES}

Biber, M. (2006) IPv6 and Australia: The Way Forward, Presented at the $2^{\text {nd }}$ IPv6 Summit, Canberra, 5-6 December.

CIO Council (2006) IPv6 Transition Guidance, Available: www.cio.gov/documents/IPv6_Transition_Guidance.doc, Accessed: 25 June 2007.

CIOL (2004) Maran's 10-point agenda for Indian IT, Available: www.ciol.com/content/news/2004/104052609.asp, Accessed: 21 June 2007.

Cooper, M. \& Yen, D.C. (2005) IPv6: business applications and implementation concerns, Computer Standards and Interfaces, Vol. 28, pp. 27-41.

Dale, P. (2006) Preparing for the Implementation of IPv6 in the Australian Government, Presented at the $2^{\text {nd }}$ IPv6 Summit, Canberra, 5-6 December.

Esaki, H. (2006) IPv6 Deployment Status in Japan: Exploring New Business Area, Presented at the $2^{\text {nd }}$ IPv6 Summit, Canberra, 5-6 December.

Hardin, G. (1968) The Tragedy of the Commons, Science, Vol. 162, pp. 1243-1248.

Hill, T. (2006) Stoking the IPv6 Debate, Information Age, October, pp. 32-34.

Hovav, A. Patnayakuni, R. \& Schuff, D. (2004) A model of Internet standards adoption: the case of IPv6, Information Systems Journal, Vol. 14, pp. 265-294.

Hovav, A. \& Schuff, D. (2005) The Changing Dynamic of the Internet: Early and Late Adopters of the IPv6 Standard, Communications of the AIS, Vol. 15, pp. 242-262.

Huston, G. (2006) IPv6 - Extinction, Evolution, or Revolution?, The ISP Column, Available: www.potaroo.net/ispcol/2006-01/ipv6revolution.pdf, Accessed: 6 June 2007.

IPv6 Task Force (2005) IPv6 Roadmap Recommendations: Internet for Everyone, Available: www.ipv6tf.org/PublicDocuments/Press_Release_IPv6TF.pdf, Accessed: 21 June 2007.

JPNIC (2006) Analysis and Recommendations on the Exhaustion of IPv4 Address Space, Japan Network Information Centre, Available: www.nic.ad.jp/en/research/IPv4exhaustion_trans-pub.pdf, Accessed: 25 June 2007.

Juniper (2006) 2nd Annual IPv6 U.S. Government Action Study: Progress and Promise of the U.S. IPv6 Transition, Juniper Networks, Available: www.juniperipv6.com, Accessed: 25 June 2007.

Kirkpatrick, D. (2006) Experts say U.S. must act on Internet, Fortune, November 6. 
Kwong, C. (2006) How Mature is IPv6?, Masters Thesis, School of Information Systems, Curtin University of Technology, Perth, Australia.

Ladid, L. (2006) IPv6 Forum Roadmap and Vision, IPv6 Forum, Available: www.ipv6forum.com/d1/forum/wwc_ipv6forum_roadmap_vision_2010.pdf, Accessed: 26 June 2007.

Liebowitz, S.J. \& Margolis, S.E. (1994) Network Externality: An Uncommon Tragedy, Journal of Economic Perspectives, Vol. 8, No. 2, pp. 133-150.

Liu, Y. (2006) What is Total Cost of Ownership of NAT?, Masters Thesis, School of Information Systems, Curtin University of Technology, Perth, Australia.

Loshin, P. (2004) IPv6: Theory, Protocol, and Practice ( $2^{\text {nd }}$ Edn), Morgan Kaufmann Publishers, USA.

NTIA (2004) Technical and Economic Assessment of Internet Protocol Version 6 $(I P v 6)$, www.ntia.doc.gov/ntiahome/ntiageneral/ipv6/draft/discussiondraftv13_0716200 4.pdf, Accessed 6 June 2007.

Richards, D. (2006) Australian Department of Defence's Transition to IPv6, Presented at the $2^{\text {nd }}$ IPv6 Summit, Canberra, 5-6 December.

Strauss, H. (2005) U.S. Government's Move to IPv6 Will Require Disciplined Implementation, Industry Research Report ID Number G00136319, Gartner.

Tanenbaum, A.S. (1996) Computer Networks ( ${ }^{\text {rd }}$ Edn), Prentice-Hall, Upper Saddle River, New Jersey.

Tatipamula, M., Grossetete, P. \& Esaki, H. (2004) IPv6 Integration and Coexistence Strategies for Next-Generation Networks, IEEE Communications, Vol. 42, No. 1, pp. 88-96. 